\title{
JABFP Listens and Sets Research Agenda
}

\author{
Anne Victoria Neale, PhD, MPH, and Kendra Schwartz, MD, MSPH
}

Primary care researchers gathered in record numbers at the annual meeting of the North American Primary Care Research Group (NAPCRG), held in October 2003 in Alberta, Canada. The conference theme of "Collaboration" was evident from the many different presentations coming from practice-based research networks, cancer researchers, and those exploring the methods of complexity science. The plenary and keynote speakers also considered the importance of collaboration in their past successes, while stimulating discussion about the role of collaboration in the future directions of primary care research.

Michael C. Klein, MD, CCFP, recipient of the 2003 Maurice Wood Award for Lifetime Contribution to Primary Care Research, gave a fascinating overview of his career, highlighting important collaborations. His diverse training in medical anthropology, pediatrics, neonatology, community medicine, and family practice has allowed him to address primary care problems with multiple perspectives. From a single patient with lead poisoning, he determined that the source of contamination was a ceramic pitcher with a lead-based glaze frequently used at family meals. Dr. Klein recognized the enormous public health implications and started an awareness campaign that resulted in the prohibition of lead-based glazes in vessels intended for food use.

Dr. Klein also described his maternity care work that led him to challenge the conventional wisdom on the need for routine episiotomy. His famous "episiotomy trial" was a randomized study demonstrating that women given episiotomies during deliveries were more likely to experience severe perineal lacerations and greater pain than women who

Submitted 18 Nov 2003.

From the Department of Family Medicine, Division of Practice-based Research, Wayne State University School of Medicine, Detroit (AVN, KS). Address correspondence to Dr. Victoria Neale, Department of Family Medicine, 101 E. Alexandrine, Detroit, MI, 48201 (e-mail: vneale@ med.wayne.edu). did not receive this procedure. This study was instrumental in decreasing the use of routine episiotomy.

Allen Dietrich, MD, delivered a plenary talk that also highlighted his collaborative career. From a Zuni Native American community, he learned the importance of respecting cultural traditions and concerns in the effort to develop a continuity of care approach. He then led a community outreach effort in rural Alaska to provide cervical cancer screening to women who had little or no opportunity to receive Papanicolaou tests. This effort involved training local women to recruit patients and deliver their preventive care.

In recognition of his record of community and cross-discipline collaboration, Dr. Dietrich was recently asked to lead the MacArthur Initiative on Depression and Primary Care, a major effort to apply evidence-based knowledge to improve depression diagnosis and treatment in primary care settings. He is working with psychiatrists, internists, health policy makers, and other family physicians to develop novel systems of depression care.

Brian Hennen, MD, MA, Dean of the Faculty of Medicine at the University of Manitoba, discussed a model to increase collaboration in academic settings, considering in turn points of view from the Dean's office, from the chairs of clinical departments, and from faculty clinicians and educators. $\mathrm{He}$ reminded NAPCRG attendees that research collaborations are most successful when they demonstrate their value in improving curricula, raising standards of patient care, and providing benefits to both researchers and sponsors. He also expressed concern that an undue emphasis on randomized clinical trials was stifling researcher creativity, and he challenged journals to provide other avenues of exploration, including the opportunity to publish clinical observations, encourage new research models, and promote dialogue with scientists and practitioners from other disciplines. Quoting sociobiologist E. O. Wilson that "the greatest enterprise of the mind has always been, and will always be, in 
the attempted linkage of the sciences and humanities," he encouraged the audience to recognize the benefit of including perspectives from the humanities to improve both their research and their patient care.

Iona Heath, BChir, MRCP, CBE, challenged primary care researchers to rethink their research agenda. She noted the striking inverse relationship between the health care budget for a given population and the self-rated health of the same population, contrasting developed and developing countries. People in countries with higher life expectancies reported poorer perceived health compared with people in countries with lower life expectancies. She proposed that corporate profit interests underpin trends that shift attention and investment within health care from the sick to the well, from the poor to the rich, and from old to the young, allowing care mediated by touch to be displaced by systems driven by paper and computers.

Dr. Heath posited that the focus in developed countries on screening and prevention has resulted in the medicalization of life for the healthy majority. The emphasis on reducing risk factors and searching for predisposing factors for disease among the healthy ("disease mongering") has the effect of redirecting care away from the needs of the sick minority. Dr. Heath challenged practitioners and researchers to address the sick minority and seek to reduce socioeconomic inequalities in health. This provocative talk resulted in some reappraisal of the preventive services research presented at the meeting.

The $7 A B F P$ will respond to the challenges issued by these thoughtful primary care leaders. We will inaugurate a new feature that recognizes the importance of the humanities in medicine. The Center for Ethics and Humanities in the Life Sciences at Michigan State University will develop and edit this feature. Center faculty will consider and respond to selected ethical dilemmas submitted by readers. In addition, authors are invited to submit original research related to clinical, research, or publication ethics.

To further promote collaborative research efforts, we invite submissions from established teams, such as practice-based research networks, and work that demonstrates novel and creative methods that emerge from interdisciplinary work groups. In addition, the $7 A B F P$ is very interested in receiving submissions that report on programs or studies that address health inequities among the sick, the aged, and the poor. Patient-oriented outcome studies are especially welcomed. Cancer research was emphasized at the NAPCRG meeting, and we encourage reports of efforts to improve the primary care of patients with cancer and reports that describe the survivorship experience.

To provide a forum for clinical observations, and other important perspectives, $7 A B F P$ will continue to publish Brief Reports. This feature includes original pilot studies and case reports that offer novel clinical observations and discussions. These should emphasize a teaching point of clinical relevance or offer a perspective related to improving clinical practice or research.

Finally, the $7 A B F P$ seeks to collaborate with the best primary care researchers. First, we invite you to submit your best work for publication consideration. Because of our large circulation and the free on-line availability of full-text articles, your work will be widely distributed to primary care clinicians and researchers, with great potential to decrease the time lag between discovery and implementation. Second, we are seeking peer reviewers with critical appraisal skills who can identify those submissions with the greatest potential to improve primary care research and practice. Through careful deliberation and constructive critique, peer reviewers also have a vital role in building the skills of researchers and authors, as well as advancing the field. Our volunteer peer reviewer form is available on our web site at http://www.jabfp.org. 